\title{
Simple mathematical equation to predict distance to gastro- esophageal junction through the submucosal tunnel during peroral endoscopic myotomy
}

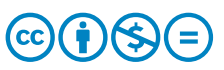

\author{
Authors \\ Jay Bapaye ${ }^{1,2}$, Tarun Bharadwaj ${ }^{1}$, Amol Bapaye ${ }^{1}$, Mahesh Mahadik ${ }^{1}$, Rajendra Pujari ${ }^{1}$, Suhas Date ${ }^{1}$, Sravan Kumar \\ Korrapati ${ }^{1}$, Rishabh Jain ${ }^{1}$, Nachiket Dubale ${ }^{1}$
}

Institutions

1 Shivanand Desai Center for Digestive Disorders, Deenanath Mangeshkar Hospital and Research Center, Pune, India

2 Sinhagad Institute's Smt. Kashibai Navale Medical College, Pune, India

submitted 4.11 .2018

accepted after revision 13.5.2019

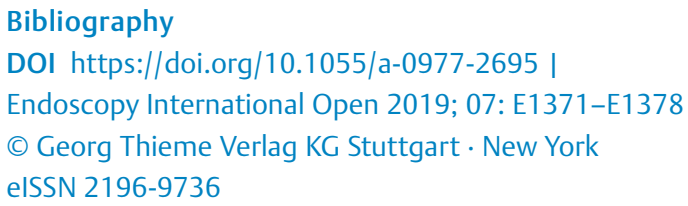

Corresponding author

Amol Bapaye, MD, FASGE, Shivanand Desai Center for Digestive Disorders, Deenanath Mangeshkar Hospital and Research Center, Pune 411004, India

Fax: +91-20-49153218

amolbapaye@gmail.com

\section{ABSTRACT}

Background and aim Accurate estimation of the distance to the gastroesophageal junction (GEJ) through a tunnel during per oral endoscopic myotomy (POEM) is technically challenging. The methods currently employed are often in- sufficient, and resultant errors may lead to incomplete myotomy and/or prolonged procedure times. Our hypothesis states that the additional distance while traversing the tunnel is directly proportional to the widest esophageal diameter; and is calculated by the formula $X=Y+C Z(X=$ distance to the GEJ through the tunnel, $Y=$ distance to the $G E J$ through the lumen, $\mathrm{Z}=$ widest esophageal diameter, $\mathrm{C}=\mathrm{ar}$ ithmetic constant). This study evaluates the validity and accuracy of this hypothesis.

Patients and methods This was a prospective single-center study with 80 patients, 12 in the pilot group and 68 in the study group. In the pilot group, $Y$ was recorded during esophagogastroduodenoscopy (EGD) before POEM, Z on barium swallow/contrast enhanced computed tomography (CECT), and X was measured during POEM. Using the formula, mean C (SD) was calculated. In the study group, ' $C$ ' was substituted in the equation to predict the GEJ distance through the tunnel $(X p)$ before POEM. The operator was blinded to $X p$ and recorded the true $X t$ during POEM. The correlation between $\mathrm{Xp}$ and $\mathrm{Xt}$ was calculated.

Results In the pilot group, the mean distances (cm, SD) for $X, Y$, and $Z$ were 42.58 (3.33), 39.83 (3.08), and 4.39 (1.16), respectively. The calculated mean $C$ was $0.63(0.11)$. In the study group, the mean distances (cm, SD) for Y, Z, Xp, and Xt were 40.45 (2.58), 4.99 (1.43), 43.57 (2.68), and 43.54 (2.78), respectively. The $\mathrm{Xp}$ and $\mathrm{Xt}$ values demonstrated a high correlation $(r=0.97, P=0.000)$.

Conclusions Formula $\mathrm{X}=\mathrm{Y}+\mathrm{CZ}$ reliably predicts the $\mathrm{GEJ}$ distance through a tunnel during POEM. It is user friendly and requires no additional resources.

\section{Introduction}

Per-oral endoscopic myotomy (POEM) is an accepted treatment modality for achalasia cardia [1-3]. POEM has been shown to have high long-term success rates up to 2 years and is also effective in patients with recurrent achalasia [4]. The modality is gaining rapid acceptance globally and the number of centers performing POEM has increased dramatically [2]. The procedure is technically demanding and requires significant endo- scopic skills in addition to an understanding of the anatomy. The learning curve for POEM is estimated to be 20 to 40 procedures [5,6].

The technique for POEM involves making a mucosal incision in the mid-esophagus approximately 8 to $10 \mathrm{~cm}$ proximal to the gastroesophageal junction (GEJ). A submucosal (SM) tunnel is created extending beyond the GEJ. Subsequent circular or fullthickness myotomy is then performed starting 2 to $3 \mathrm{~cm}$ distal to the mucosal incision and extending across the GEJ. The mu- 
cosal incision is finally closed using endoscopic clips. Both anterior and posterior approaches have been described $[1,7,8]$.

Reliable identification of the GEJ is an important technical step during POEM. Six landmarks have been reported to date to aid its identification [1-3]: (1) endoscopic measurements from the incisors; (2) initial narrowing of the submucosal space at the level of the GEJ with increased resistance followed by prompt expansion of the submucosal space beyond the gastric cardia, along with increased vascularity and "spindle"-shaped veins; (3) visualization of palisading vessels on the mucosal undersurface; (4) large-caliber, perforating vessels in the cardia representing branches of the left gastric artery; (5) visualization of aberrant longitudinal muscle bundles at the GEJ; and/or (6) visualization of a blue hue on intraluminal inspection of the mucosa of the gastric cardia on retroflexion of the endoscope in the stomach (caused by the blue dye in the injectate).

However, despite these landmarks, reliable identification of the GEJ can often remain a challenge during POEM. In patients with a history of prior interventions, especially prior balloon dilation, botulinum toxin injection or surgery, the abovementioned landmarks in the submucosal space may be obscured due to fibrosis. Endoscopists are often required to withdraw the endoscope into the esophageal lumen to observe the bluish submucosal hue in the gastric fundus to confirm adequacy of the tunnel. This step may need to be repeated more than once when GEJ identification during tunneling is in doubt. This significantly adds to overall procedure time. Inadequate length of the tunnel may result in an incomplete myotomy thereby increasing the risk of post-POEM recurrence; whereas overextension of the tunnel on the gastric side may result in an excessive myotomy resulting in a higher risk of perforation and bleeding [9]; or although unproven in clinical trials, may hypothetically predispose the patient to development of post-POEM gastroesophageal reflux disease (GERD).

Several additional techniques have been described to aid GEJ identification during POEM: injection of indocyanine green (ICG) at the cardia on retroflexion [10], double endoscope trans-illumination technique $[9,11,12]$, and use of fluoroscopy [13]. EndoFLIP has also been used to evaluate the GEJ during and after POEM $[14,15]$. Although promising, these techniques either require special equipment or set-up, are expensive or could prove logistically difficult to implement in the endoscopy suite during POEM.

In this study, we report the concept and validation results of a simple mathematical tool that can be used to predict the GEJ distance during tunneling for POEM.

\section{Hypothesis}

It has been observed that, during POEM, there is a difference between the endoscopic distance to the GEJ when measured through the esophageal lumen and through the tunnel; and that the distance through the tunnel is longer than that through the lumen. This discrepancy is pronounced in patients with sigmoid achalasia where the esophagus is grossly dilated [16], but is also seen to a variable degree in non-sigmoid patients. This difference occurs because the scope must traverse

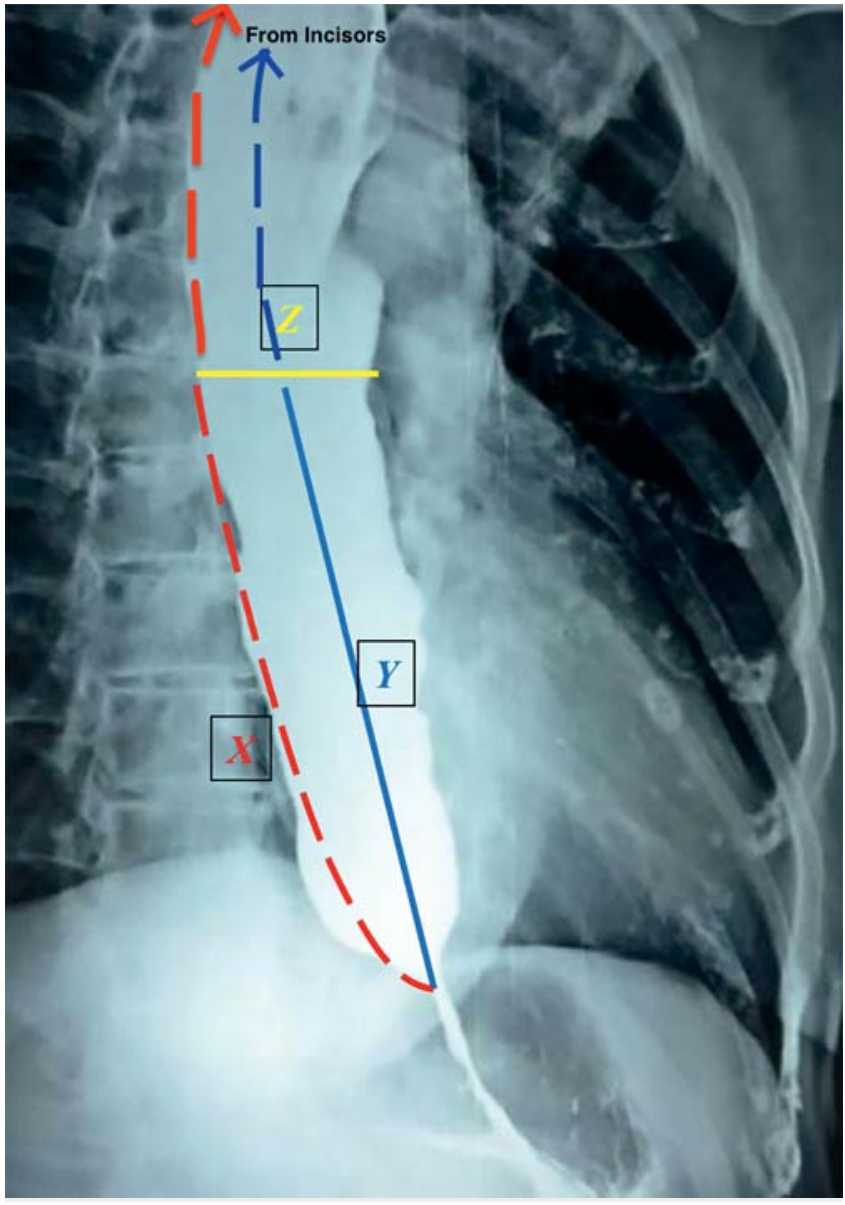

- Fig. 1 Barium esophagogram showing a dilated esophagus in a patient with achalasia cardia with measurements $X$ (distance to the gastroesophageal junction through the submucosal tunnel), $\mathrm{Y}$ (distance to the gastroesophageal junction through the esophageal lumen), and $Z$ (widest diameter of the esophagus).

a somewhat longer distance through the tunnel compared to through the lumen as it takes a curved path to reach the same end point ( $\mathbf{F i g} \mathbf{1}$ ). Therefore, the wider the proximal dilatation of the esophagus, the longer the distance that the endoscope must traverse to reach the GEJ ( $\triangleright$ Fig. 2).

To explain this in mathematical terms, let us assume that $Y$ is the distance traversed by the endoscope to the GEJ through the esophageal lumen; $X$ is the distance traversed through the SM tunnel; and $Z$ is the transverse diameter of the esophagus at its widest portion ( $\mathbf{F i g . 1}$ ). The additional distance traversed through the tunnel is the difference between $X$ and $Y$ and can be represented as $d X$. As $Z$ increases, $d X$ increases proportionately ( $\triangleright$ Fig.2). Mathematically, this equation can be written as:

$\mathrm{dX} / \mathrm{Z}=\mathrm{C}$, where ' $\mathrm{C}$ ' represents a mathematical constant.

Now since $d X$ is the difference between $X$ and $Y, d X=(X-Y)$.

Therefore, substituting $d X$ by $(X-Y)$ in the equation, the equation reads:

$(\mathrm{X}-\mathrm{Y}) / \mathrm{Z}=\mathrm{C}$ or $\mathrm{X}=\mathrm{Y}+\mathrm{CZ}$,

where $X$ is the estimated distance to the GEJ through the SM tunnel, $Y$ is the distance to the GEJ through the esophageal lu- 


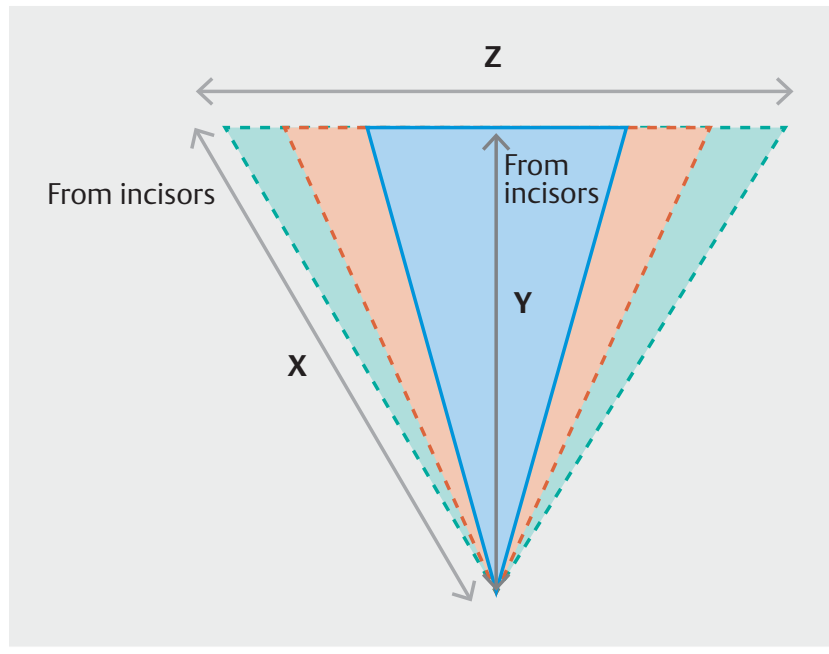

- Fig. 2 Schematic representation of $X, Y$, and $Z$ showing the proportionate increase in $\mathrm{X}$ with an increase in $\mathrm{Z}$ when $\mathrm{Y}$ is constant.

men, $\mathrm{Z}$ is the maximal esophageal diameter, and $\mathrm{C}$ is an arithmetic constant.

$Y$ can be easily calculated by esophagogastroduodenoscopy (EGD) during POEM and $Z$ by barium swallow or contrast enhanced computed tomography (CECT). If one can reliably calculate the constant $C$ for a dataset and prove that its value remains stable over a wide dataset, one can reliably predict the value of $X$ from this equation. This prediction or estimation can be of value to the endoscopist to judge the adequacy of tunneling during POEM.

The aim of this study was to validate this equation and to preoperatively predict the value of $X$ (distance to the GE) through the submucosal tunnel) up to an accuracy of within $1 \mathrm{~cm}$ of the observed value.

\section{Patients and methods}

Consecutive patients with achalasia undergoing POEM were enrolled in the study. All patients underwent EGD, high resolution manometry (HRM) and barium swallow or CECT as part of prePOEM evaluation.

Calculation of ' $Z$ ': Barium swallow or CECT films were reviewed and the widest diameter of the esophagus (Z) was recorded. While reviewing barium swallow images, the width of the thoracic vertebra was taken as a reference measurement to correct for potential magnification errors. The presence of sigmoid achalasia was recorded separately.

Calculation of ' $Y$ ': The endoscopic distance to the GEJ ( $\mathrm{Y}$ ) was measured by the endoscopist during EGD under general anesthesia before commencing POEM. All POEM procedures were performed by a single operator using the posterior approach. After introducing the endoscope via the esophagus into the stomach, the endoscope was withdrawn across the GEJ into the esophagus. Maintaining a straight endoscope, the endoscopist recorded the GEJ distance $(Y)$.

Calculation of ' $X$ ': POEM was performed using the standard four-step technique: SM elevation and incision, SM tunneling,

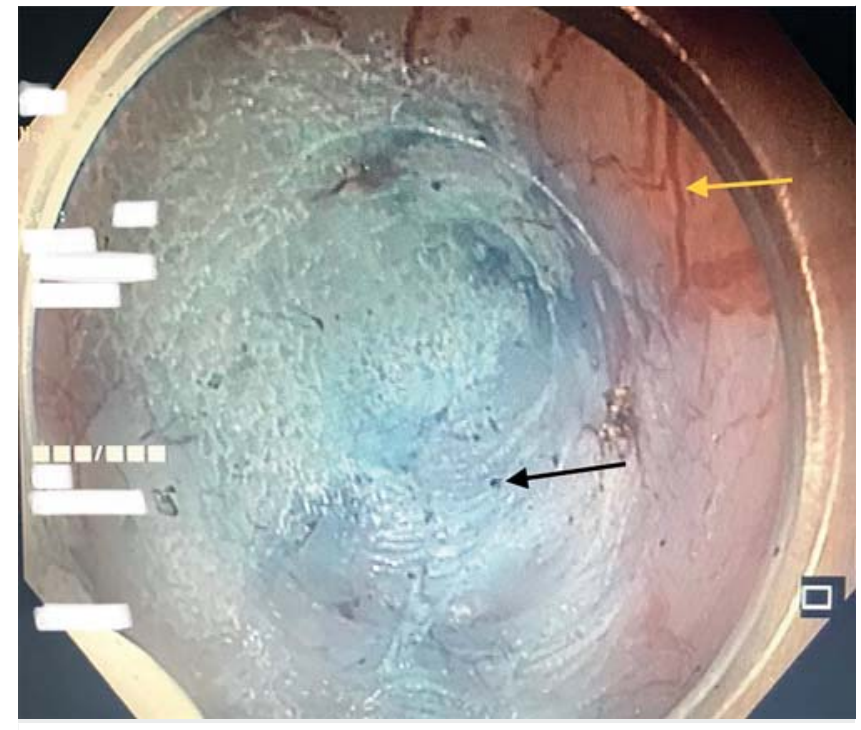

- Fig. 3 Endoscopic image of the GEJ through the submucosal tunnel. Note the palisading vessels on the mucosal aspect (yellow arrow) and spindle-shaped vessels on the muscle layer (black arrow). Also note the narrow submucosal space at the GEJ.

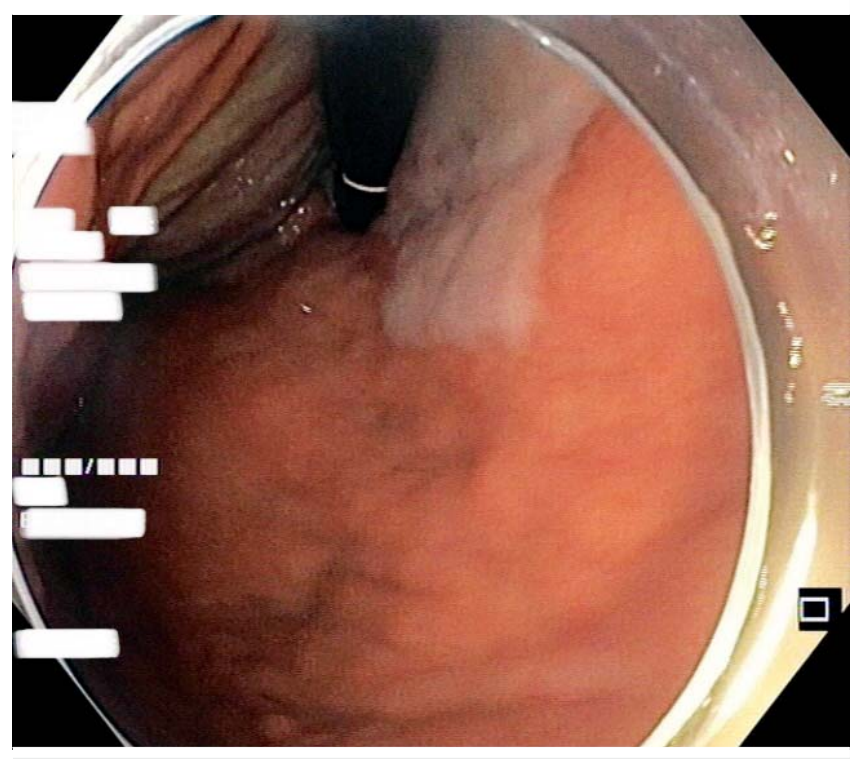

- Fig. 4 Retroflexed endoscopic image of the gastric cardia after completion of the submucosal tunnel on the gastric side. Note the bluish discoloration of the gastric mucosa at the cardia.

myotomy, and mucosal closure $[1,2]$. After mucosal incision, SM tunneling was continued in a direction perpendicular to the circular muscle fibers up to the GEJ until the standard landmarks were identified: initial narrowing of the submucosal space at the level of the GEJ with increased resistance followed by its sudden expansion; and visualization of "spindle"-shaped veins in the SM layer and palisading vessels on the mucosal undersurface ( $\vee$ Fig. 3 ). At this point, the operator measured the endoscopic distance, again maintaining the endoscope in a straight position. This distance $X$ was recorded. The endoscope was withdrawn from the tunnel and the GEJ was inspected on 
- Table 1 Patient characteristics and specifics of procedure and adverse outcomes.

\begin{tabular}{|c|c|c|c|c|}
\hline & Pilot group $(n=12)$ & Study group $(n=68)$ & Total $(n=80)$ & $P$ value \\
\hline Age, mean (range), years & $40.41(17-75)$ & $41(12-83)$ & $40.7(12-83)$ & 0.46 \\
\hline Male/female & $4: 8$ & $35: 33$ & $39: 41$ & 0.35 \\
\hline Procedure time, mean (range), min & $124(60-180)$ & $96(40-270)$ & $110(40-270)$ & 0.67 \\
\hline No. of clips for closure, $\mathrm{n}$ (range) & $8.75(6-20)$ & $6.5(5-11)$ & $7.62(5-20)$ & 0.15 \\
\hline Technical success, \% & 100 & 100 & 100 & n.s. \\
\hline Clinical success, \% & 100 & 100 & 100 & n.s. \\
\hline Adverse events, $\mathrm{n}(\%)$ & $2(17)$ & $14(21)$ & $16(20)$ & 1.000 \\
\hline - Mucosal injury & 2 & 3 & & \\
\hline - Subcutaneous emphysema & & 8 & & \\
\hline - Tension capnoperitoneum & & 3 & & \\
\hline Post POEM GERD, n (\%) & $3(25)$ & $10(14.7)$ & $13(16.3)$ & 0.4 \\
\hline Previous therapy, $\mathrm{n}(\%)$ & $4(33.3)$ & $4(5.9)$ & $8(10)$ & 0.015 \\
\hline - Pneumatic dilatation & 3 & 1 & & \\
\hline - Heller's myotomy & 1 & 3 & & \\
\hline Achalasia type (I / II / III) & $2 / 9 / 1$ & $3 / 59 / 6$ & & 0.16 \\
\hline Sigmoid esophagus, $\mathrm{n}$ & 4 & 7 & 11 & 0.06 \\
\hline
\end{tabular}

the luminal side to confirm dissection beyond the GEJ. SM dissection was further performed for an additional 2 to $3 \mathrm{~cm}$ to complete the tunnel on the gastric side ( $\triangleright$ Fig. 4 ). Subsequent POEM was then completed - full-thickness myotomy was performed in all patients and the mucosal incision was closed using clips.

The study comprised two parts: part I (pilot group) - calculation of ' $C$ ' constant (initial 12 patients), and part II (study group) - prospective estimation and measurement of predicted $\mathrm{Xp}$ and true $\mathrm{Xt}$ and validation of the hypothesis by comparing $X p$ and $X t$ (68 patients).

\section{Part I-Pilot group}

During POEM, distance to the GEJ through the tunnel was measured by the operator and was recorded. X, Y, and $Z$ values for each of the 12 patients were tabulated. These values were substituted in the equation $X=Y+C Z$ and $C$ was calculated for each record $(C=(X-Y) / Z)$. Mean $C( \pm S D)$ was calculated from the database.

\section{Part II - Study group}

In these subsequent 68 patients, $Z$ values were recorded during screening investigations. $Y$ was calculated during EGD under general anesthesia. The value of $X$ was predicted before POEM using the formula $X p=Y+C Z$, using the mean $C$ value derived from the pilot group. The endoscopist performing POEM was blinded to this Xp value. During tunneling for POEM, the endoscopist measured the true distance, $\mathrm{Xt}$, and this was recorded. $X p$ and $X t$ values were compared for each patient. An additional note was made if the difference between $\mathrm{Xp}$ and $\mathrm{Xt}$ exceeded 1 $\mathrm{cm}$.

\section{Statistical methods}

The paired $t$ test was used for continuous variables and $\mathrm{Fi}$ scher's exact test was used for categorical variables. Pearson's correlation coefficient ' $r$ ' was used to determine the strength of the association between $\mathrm{Xp}$ and $\mathrm{Xt}$, and the paired $t$ test was employed to determine significance. Spearman's correlation coefficient and Wilcoxon signed-rank test were used for subgroup analysis of patients with sigmoid achalasia. A $P$ value less than 0.05 was considered statistically significant. All statistical analysis was performed using SPSS software Ver. 20 (IBM Corp., Armonk, NY, USA).

\section{Results}

In total, 80 patients were enrolled in the study. Patient characteristics and details of type of achalasia are included in $>\mathbf{T a}$ ble 1. Patient characteristics and achalasia types were comparable in both groups $(P>0.05)$, apart from history of prior therapy, which was more frequent in the pilot group $(33 \%, P=$ $0.015)$. Mean age was 40.7 years; 39 were male. Five patients had type I, 68 had type II, and 7 had type III achalasia. Eleven (13.7\%) patients had sigmoid achalasia; 72 (90\%) patients were treatment naïve, whereas $8(10 \%)$ had a history of prior therapy.

POEM was technically successful in all 80 patients (100\%). According to the ASGE Lexicon [17], no severe adverse events 
- Table 2 Pilot group $(n=12)$ - distance measurements and calculation of constant ' $C$ '.

\begin{tabular}{|c|c|c|c|c|}
\hline Patient no. & $\mathrm{X}, \mathrm{cm}$ & $\mathrm{Y}, \mathrm{cm}$ & $\mathrm{Z}, \mathrm{cm}$ & C \\
\hline 1 & 46 & 44 & 3.75 & 0.53 \\
\hline 2 & 42 & 39 & 3.75 & 0.80 \\
\hline 3 & 45 & 41 & 6.25 & 0.64 \\
\hline 4 & 48 & 44 & 6.25 & 0.64 \\
\hline 5 & 40 & 38 & 4.81 & 0.42 \\
\hline 6 & 44 & 41 & 4.46 & 0.67 \\
\hline 7 & 38 & 36 & 3.00 & 0.67 \\
\hline 8 & 47 & 45 & 4.38 & 0.46 \\
\hline 9 & 43 & 39 & 5.83 & 0.69 \\
\hline 10 & 41 & 39 & 3.13 & 0.64 \\
\hline 11 & 39 & 36 & 4.25 & 0.71 \\
\hline 12 & 38 & 36 & 2.78 & 0.72 \\
\hline Mean & 42.58 & 39.83 & 4.39 & 0.63 \\
\hline SD & & & & \pm 0.11 \\
\hline
\end{tabular}

were recorded. Minor adverse events occurred in 16 patients (20\%): self-resolving subcutaneous emphysema in 8 , tension capnoperitoneum in 3 and small inadvertent mucosotomy in 5 patients. No additional intervention was necessary for patients with subcutaneous emphysema. Tension capnoperitoneum was treated by abdominal paracentesis in all three patients. Mucosotomy was treated by application of one or two mucosal clips. Asymptomatic capnoperitoneum and capnomediastinum were not recorded as adverse events. No major adverse events were encountered.

The pilot group consisted of the initial 12 patients ( $\triangleright$ Table2). Sigmoid achalasia was encountered in 4 (33.3\%) patients. Mean values (SD) for $\mathrm{X}, \mathrm{Y}$, and $\mathrm{Z}$ in the pilot group were $42.58 \mathrm{~cm}(3.33), 39.83 \mathrm{~cm}(3.08)$, and $4.39 \mathrm{~cm}(1.16)$, respectively. Mean $C(S D)$ calculated using the formula $X=Y$ + CZ was 0.63 (0.11) ( Fig.5).

The study group consisted of the subsequent 68 patients ( $\downarrow$ Table 3). Sigmoid achalasia was encountered in 7 (10\%) patients. Mean $Y$ in the study group was $40.45 \mathrm{~cm}$ (2.58), and mean Z was $4.99 \mathrm{~cm}(1.43)$. Mean C (calculated from the pilot study data) was 0.63 . Mean predicted Xp was $43.57 \mathrm{~cm}$ (2.68) and mean measured $X t$ was $43.54 \mathrm{~cm}$ (2.78). Xp and $X t$ values demonstrated an extremely high correlation (Pearson's correlation coefficient $r=0.97, P=0.000)$. In $62 / 68$ (91.2\%) patients, the difference between $\mathrm{Xp}$ and $\mathrm{Xt}$ was less than $1 \mathrm{~cm}$ ( $\triangleright$ Fig. 6). Of the six patients, wherein the difference between $\mathrm{Xp}$ and $\mathrm{Xt}$ was greater than $1 \mathrm{~cm}$, no significant differences in the age group, gender, and achalasia subtype were noted. Four patients were female, ages ranging from 12 to 58 years; and five pa-

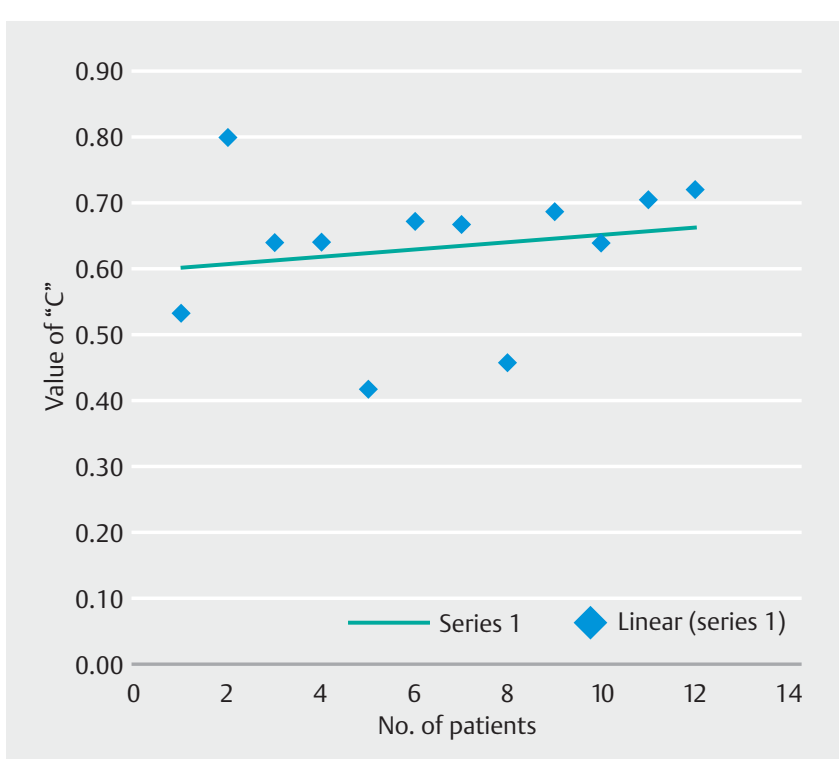

- Fig. 5 Scatter plot for constant ' $C$ '. The graph shows that the majority of values are close to the mean.

tients had type II achalasia whereas one had type I. Subgroup analysis for sigmoid achalasia showed that the correlation was maintained at the same level of significance (Spearman's correlation coefficient $r=0.973, P=0.000)$ ( $\triangleright$ Table 4$)$.

\section{Discussion}

The POEM technique is challenging and a learning curve of about 20 to 40 procedures has been proposed [5,6]. Several steps in POEM require a clear and detailed understanding of the submucosal and mediastinal anatomy, the layers of the esophagus and stomach, and their appearance and vasculature. A miscalculation or misjudgment during any of these procedural steps may compromise the safety and/or efficacy of the procedure. Reliable identification of the GEJ is an important step during POEM, since the SM tunnel must cross the GEJ into the stomach for an optimal result.

Several anatomical landmarks for reliable GEJ identification have been described [1-3]. However, being anatomical landmarks, a change in anatomy can alter these landmarks and they may no longer be appreciable during the procedure. SM vascular patterns such as palisading and spindle-shaped vessels are especially likely to get distorted in a postoperative, post-Botox injection or post-dilatation anatomy. In sigmoid achalasia, the endoscopic GEJ distance may be fallaciously farther away due to looping of the endoscope. Additional objective measures - use of fluoroscopy, ICG injection, double endoscope trans-illumination or EndoFLIP have therefore been introduced to supplement anatomical findings; however, these additional measures require specialized equipment or set-up, and may therefore increase procedure cost or may have logistical difficulties in implementation.

This study describes a mathematical method to predict the GEJ during tunneling for POEM. The method is based upon the 
- Table 3 Correlation of mean $( \pm S D)$ predicted $X p$ and mean true $X t$ in the study group $(n=68)$.

\begin{tabular}{|c|c|c|c|c|c|c|}
\hline & $\mathrm{Y}, \mathrm{cm}$ & $\mathrm{Z}, \mathrm{cm}$ & $\mathrm{Xp}, \mathrm{cm}$ & $\mathrm{Xt}, \mathrm{cm}$ & Pearson correlation coefficient & $P$ value \\
\hline Mean & 40.45 & 4.99 & 43.57 & 43.54 & 0.97 & 0.000 \\
\hline SD & \pm 2.58 & \pm 1.43 & \pm 2.68 & \pm 2.78 & & \\
\hline
\end{tabular}

GEJ, gastroesophageal junction; $X p$, predicted distance to the GEJ through the submucosal tunnel; $X p$, true distance to the GEJ through the submucosal tunnel; Y, distance to the GEJ through the lumen; $Z$, widest esophageal diameter.

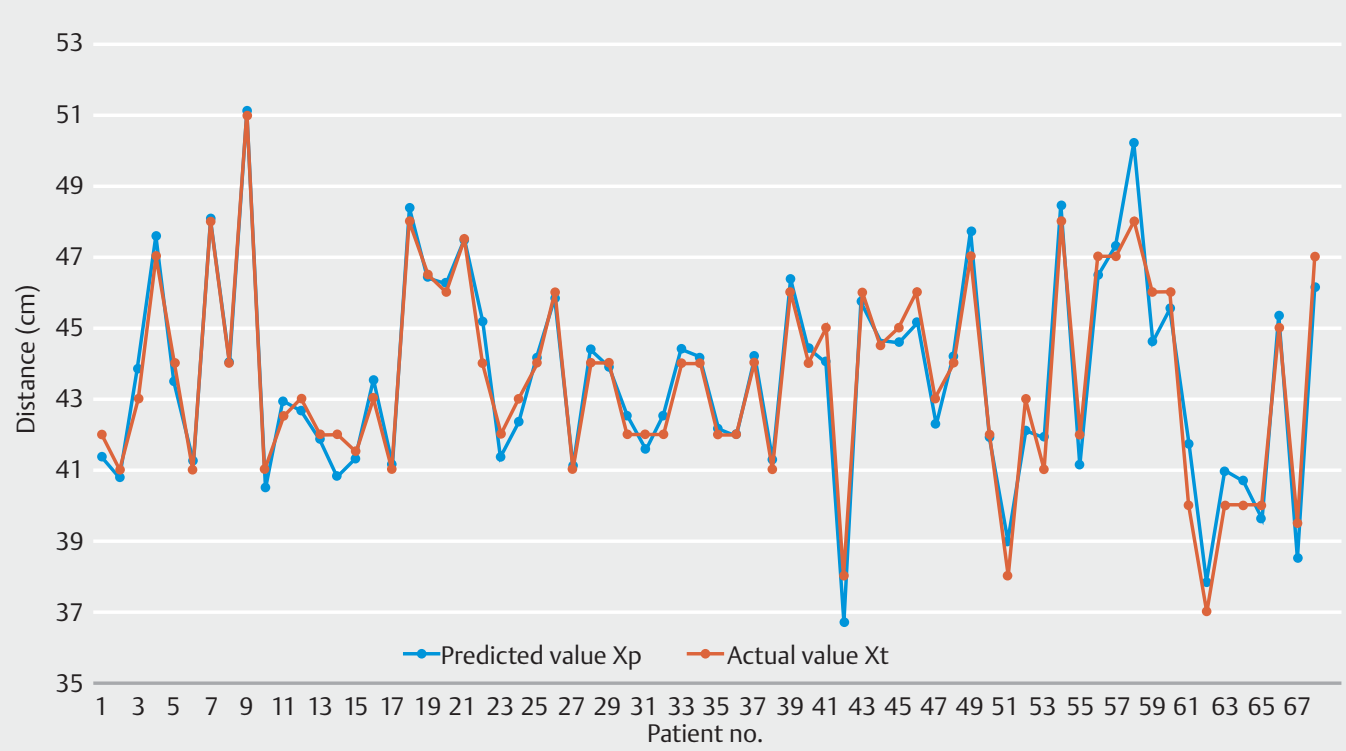

- Fig. 6 Graph showing the correlation between Xp and Xt for the 68 patients in the study group. The graph clearly depicts the close overlap of these two values for each patient.

- Table 4 Correlation between predicted Xp and true Xt in the subgroup with sigmoid achalasia $(n=7)$.

\begin{tabular}{|l|c|c|c|c|c|c|}
\hline & $\mathbf{Y}, \mathbf{c m}$ & $\mathbf{Z}, \mathbf{c m}$ & $\mathbf{X p}, \mathbf{c m}$ & $\mathbf{X t ,} \mathbf{c m}$ & Spearman correlation coefficient \\
\hline Mean & 43.15 & 7.03 & 47.57 & 47.29 & 0.973 \\
\hline SD & \pm 2.04 & \pm 1.49 & \pm 2.45 & \pm 2.05 & \\
\hline
\end{tabular}

fact that there is a discrepancy in the GEJ distance when measured through the lumen and through the tunnel. This has been reported by other authors [16]; however, the difference has never been quantified or the discrepancy has never been analyzed. This study attempts to analyze this difference and uses it to devise and validate an equation by which one can estimate the correct distance.

The results of our study demonstrate a very strong, highly significant linear relationship between predicted and actual values of $X(r=0.97, P=0.000)$. The effect is sustained even in cases of sigmoid achalasia $(r=0.973, P=0.000)$. It must be noted that the difference between $X p$ and $X t$ was less than $1 \mathrm{~cm}$ in $91.2 \%$ patients. Since the measurements on the endoscope are $1 \mathrm{~cm}$ apart, distance discrepancies under $1 \mathrm{~cm}$ have limited significance during endoscopic measurements.
In any mathematical equation, a stable and reliable value of the constant is considered important for successful application of the equation. If the constant keeps changing, the equation loses its value. In our study, values for constant $C$ demonstrated reliable stability in our pilot group. Also, when substituted in the equation in the study group, Xp and Xt demonstrated a significant and close correlation. Both of these factors demonstrate that the value $C=0.63$ can be considered to be a reliable constant.

The advantage of this method is its simplicity and the fact that no special instrumentation is needed. EGD and barium swallow are standard investigations for evaluation of most achalasia patients. Therefore, the method can be implemented in nearly every patient undergoing POEM without additional effort. In comparison, other described techniques use either 
fluoroscopy, ICG, EndoFLIP, or an additional transnasal endoscope to identify this landmark [9-11,13-15,18,19]. This can result in additional procedure costs and can create logistical difficulties to schedule and perform POEM within endoscopy or operating suites.

While performing POEM, the endoscopist has a choice of several landmarks that can be used for estimation of the GEJ. Not all landmarks are identifiable in every patient, and often more than one landmark is required for reliable confirmation. Most endoscopists would prefer to use more than one landmark. The current equation presents a simple mathematical and therefore non-anatomical tool for GEJ estimation. Given the results of our study, we believe that this non-anatomical estimation could be used in conjunction with the standard anatomical markers to further add accuracy while calculating the GE] during POEM. This may be especially useful in patients with recurrent achalasia after prior therapy, since in these patients, standard anatomical markers may be obscured due to the earlier intervention.

There are certain limitations to this study. The value of $C$ has been calculated based on the data obtained from 12 patients. Increasing the size of the database may improve the accuracy of $\mathrm{C}$ as a constant and produce more accurate estimates of the $\mathrm{GEJ}$ distance. The technique to measure the GEJ can be somewhat subjective amongst endoscopists. We recommend the technique described in the study for optimum measurements and results. It is noteworthy, however, that despite these limitations, the hypothesis and equation in this study demonstrate an accurate and reliable estimation of the GEJ through the tunnel. This is possibly because the deviations in values due to calculation errors are small (less than $1 \mathrm{~cm}$ ) and therefore insignificant in the context of measuring the GEJ using endoscopic markings. Another potential factor for bias is the estimation and calculation of $\mathrm{Xp}$ and $\mathrm{Xt}$; however, in this study, the operating endoscopist was blinded to the value of $X p$ thereby eliminating this bias.

There are several ways to implement this equation in clinical practice. It can be used as a scouting method to identify the GEJ in situations when anatomical landmarks are obscured or when the endoscopist is unsure about the adequacy of the SM tunnel. It is obviously not designed to replace any of the prevalent anatomical landmarks, which must also be identified by the endoscopist before confirming the adequacy of the tunnel; however, an endoscopist may choose to dissect in the SM tunnel until this distance $X$ has been reached before beginning to look around for anatomical landmarks to confirm completion. This can speed up the procedure considerably. Endoscopists often have a natural tendency to dissect deep on the gastric side beyond the GEJ to safeguard against recurrence. This can increase the risk of perforation or bleeding [9]. Also, although not validated in a research protocol, there is a suggestion that a longer gastric myotomy may predispose to development of post POEM GERD. This equation may alert the endoscopist to the arrival of the GEJ and may thus reduce the risk of an inadvertently long gastric myotomy, thereby minimizing the risk of post POEM GERD.
In conclusion, this study demonstrates and validates a simple mathematical formula that can be used reliably to measure the distance to the GEJ while creating a tunnel during POEM. The formula displays a high correlation between predicted and true measurements. The technique is easy to use and does not require any additional equipment. Studies to further validate the reliability of this hypothesis and to address any further limitations are recommended.

\section{Acknowledgements}

The authors wish thank Dr. Asawari N. Kanade, clinical statistician, for her support with the statistical analysis in this study. The authors would also wish to thank Ms. Shivangi Dorwat, clinical research coordinator, for assistance with conducting the study including data collection and analysis.

\section{Competing interests}

None

\section{References}

[1] Inoue $\mathrm{H}$, Minami $\mathrm{H}$, Kobayashi Y et al. Peroral endoscopic myotomy (POEM) for esophageal achalasia. Endoscopy 2010; 42: 265-271

[2] Stavropoulos SN, Modayil RJ, Friedel D et al. The International Per Oral Endoscopic Myotomy Survey (IPOEMS): a snapshot of the global POEM experience. Surg Endosc 2013; 27: 3322 - 3338

[3] von Renteln D, Inoue H, Minami H et al. Peroral endoscopic myotomy for the treatment of achalasia: a prospective single center study. Am J Gastroenterol 2012; 107: 411-417

[4] Ngamruengphong S, Inoue H, Ujiki MB et al. Efficacy and safety of peroral endoscopic myotomy for treatment of achalasia after failed Heller myotomy. Clin Gastroenterol Hepatol 2017; 15: 1531 - 1537.e3

[5] Patel KS, Calixte R, Modayil R] et al. The light at the end of the tunnel: a single-operator learning curve analysis for per oral endoscopic myotomy. Gastrointest Endosc 2015; 81: 1181 - 1187

[6] Kurian AA, Dunst CM, Sharata A et al. Peroral endoscopic esophageal myotomy: defining the learning curve. Gastrointest Endosc 2013; 77 : $719-725$

[7] Minami H, Isomoto $\mathrm{H}$, Yamaguchi $\mathrm{N}$ et al. Peroral endoscopic myotomy for esophageal achalasia: clinical impact of 28 cases. Dig Endosc 2014; $26: 43-51$

[8] Zhou PH, Li QL, Yao LQ et al. Peroral endoscopic remyotomy for failed Heller myotomy: a prospective single-center study. Endoscopy 2013; 45: $161-166$

[9] Baldaque-Silva F, Marques M, Vilas-Boas F et al. New transillumination auxiliary technique for peroral endoscopic myotomy. Gastrointest Endosc 2014; 79: $544-545$

[10] Minami H, Inoue $\mathrm{H}$, Haji A et al. Per-oral endoscopic myotomy: emerging indications and evolving techniques. Dig Endosc 2015; 27 : $175-$ 181

[11] Grimes KL, Inoue H, Onimaru M et al. Double-scope per oral endoscopic myotomy (POEM): a prospective randomized controlled trial. Surg Endosc 2016; 30: 1344-1351

[12] Khashab MA, Kumbhari V, Azola A et al. Intraoperative determination of the adequacy of myotomy length during peroral endoscopic myotomy (POEM): the double-endoscope transillumination for extent confirmation technique (DETECT). Endoscopy 2015; 47: 925-928 
[13] Kumbhari V, Saxena P, Messallam AA et al. Fluoroscopy to document the extent of cardiomyotomy during peroral endoscopic myotomy. Endoscopy 2014; 46: (Suppl. 01): E369-370

[14] Familiari P, Gigante G, Marchese M et al. EndoFLIP system for the intraoperative evaluation of peroral endoscopic myotomy. United Eur Gastroenterol J 2014; 2: 77-83

[15] Kalapala R, Nageshwar Reddy D, Ramchandani M et al. "EndoFLIP" in assessment of esophagogastric junction distensibility prior to and during per oral endoscopic myotomy. Indian J Gastroenterol 2015; 34 : $270-272$

[16] Hu JW, Li QL, Zhou PH et al. Peroral endoscopic myotomy for advanced achalasia with sigmoid-shaped esophagus: long-term out- comes from a prospective, single-center study. Surg Endosc 2015; 29 : $2841-2850$

[17] Cotton PB, Eisen GM, Aabakken L et al. A lexicon for endoscopic adverse events: report of an ASGE workshop. Gastrointest Endosc 2010; 71: $446-454$

[18] Nabi Z, Ramchandani M, Chavan R et al. Per-oral endoscopic myotomy for achalasia cardia: outcomes in over 400 consecutive patients. Endosc Int Open 2017; 5: E331 -E339

[19] Ramchandani M, Nageshwar ReddyD, Darisetty S et al. Peroral endoscopic myotomy for achalasia cardia: Treatment analysis and followup of over 200 consecutive patients at a single center. Dig Endosc 2016; $28: 19-26$ 